\title{
The Impact of an Adapted Climbing Program on Children with Attention-Deficit/Hyperactivity Disorder
}

\author{
Erin Angelini a, Kathryn N Oriel a,*, Greta M Myers a, Kyle D.A. Cook ${ }^{\text {a, }}$ \\ Ross M. Drawbaugh a, Jennifer Price a \\ a Department of Physical Therapy, Lebanon Valley College, 101 N College Ave, Annville, PA 17003 \\ *Corresponding author Ph +55 9481700066; Email: oriel@lvc.edu \\ DOI: https://doi.org/10.34256/ijpefs2047 \\ Received: 25-03-2020, Revised: 3-12-2020; Accepted: 5-12-2020; Published: 12-12-2020
}

\begin{abstract}
Attention-Deficit/Hyperactivity Disorder impacts children's participation in activities that require attention to instruction, sustained mental effort, and executive functioning. Physical activity has been correlated to improvement in attention in children with ADHD. Rock climbing challenges muscular endurance, attention, and route planning. Five participants, aged 8-13, participated in the climbing program. Attention was measured pre and post climbing intervention with Trail Making Test B (TMT-B) for time to complete. Exercise intensity was measured by heart rate. Parent feedback on behavior was collected with the Conner's Parent Rating Scale (CPRS). The social validity of the intervention was measured by the IRP-15 measures. Statistically, significant intrasession attention improvements were noted in all 5 climbers $(p=.43)$. Two climbers were consistently working at a moderate intensity (40-60\% HRmax) while 3 climbers maintained a light level of intensity (20-40\% HRmax). No statistically significant improvements were found on the CPRS, although improvements are noted with qualitative reports from parents. The IRP-15 showed $100 \%$ of parents believed rock climbing was an effective intervention for their children with ADHD. Rock climbing at a light to moderate intensity is associated with improvements in attention and behavior in children with ADHD.
\end{abstract}

Keywords: Climbing, ADHD, Attention.

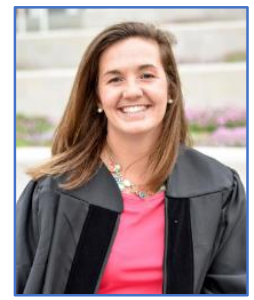

Dr. Erin Angelini, PT, DPT, is a physical therapist finishing her residency in sports medicine in Pittsburgh, PA. She has a focus on youth and high school athletes with a special interest in working with runners. Research interests include injury epidemiology and injury prevention programs in the adolescent athlete. She has presented this research with her co-authors at Combined Sections Meeting in 2019.

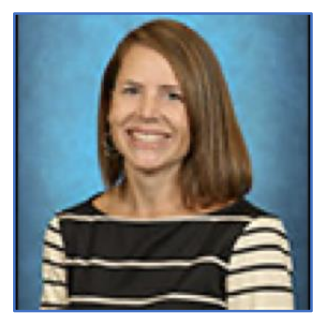

Dr. Kathryn Oriel, PT, EdD, is a professor in the department of physical therapy at Lebanon Valley College, where she currently teaches all pediatric content, along with Health Promotion for Self and Society. Dr. Oriel has developed and implemented a variety of community-based programs for children of all ages with physical and cognitive disabilities. Programs have included a variety of land and aquatic interventions. She has conducted a variety of studies testing the efficacy and social validity of these programs and has published her work in a variety of peer-reviewed journals. She also maintains a clinical practice in Early Intervention.

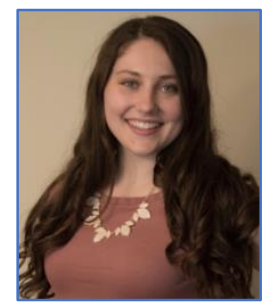

Dr. Greta (Weidemoyer) Myers, PT, DPT, graduated from Lebanon Valley College with her Doctor of Physical Therapy degree. She is a physical therapist at Lancaster Rehabilitation Hospital, where she primarily works with a neurological population. Greta has presented research at a national conference for physical therapy and a conference for student run free clinics.

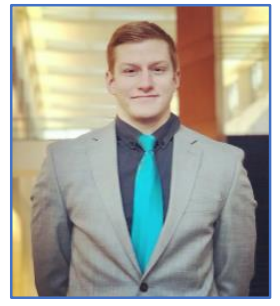

Dr. Kyle Cook, PT, DPT, is a graduate of Lebanon Valley College with degrees in Physical Therapy and Health Science. He works with Core Medical Group as a traveling physical therapist with a focus in 
outpatient orthopedics and sports rehabilitation. He has presented research at Combined Sections Meeting 2019 involving exercise and its effects on children with disabilities.

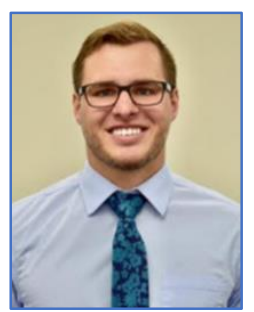

Dr. Ross Drawbaugh, PT, DPT, works in an outpatient physical therapy clinic and specializes in manual interventions. He graduated from Lebanon Valley College in 2019, where he also played for their baseball team. His love for sports and fitness began when he was young, playing five sports and that has now transitioned into a love for working with athletes in the clinic.

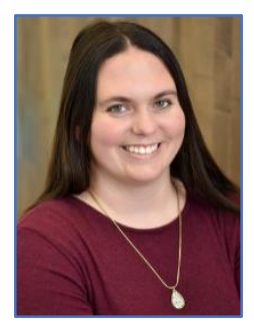

Dr. Jennifer Price, PT, DPT, Board Certified Pediatric Clinical Specialist, is the Director of Clinical Education in the department of physical therapy at Lebanon Valley College, where she currently manages the clinical experiences for 150 students. Dr. Price has participated in a variety of community-based programs for children of all ages with physical and cognitive disabilities. Programs have included a variety of land and aquatic interventions. She also maintains a clinical practice in Early Intervention.

\section{Introduction}

Attention-Deficit/Hyperactivity Disorder (ADHD) occurs in about $9.4 \%$ of children aged 2-17 years old [1]. ADHD is characterized by a delay in development of the frontal cortex, resulting in 3 major features: inattention, hyperactivity, and impulsivity. Children with ADHD present with multiple symptoms, such as being easily distracted, difficulty following instructions, avoidance of activities requiring sustained mental effort, inability to organize tasks, moving when it is not appropriate, making decisions without considering the consequences, and interrupting others [2]. As a result, children with ADHD can have difficulty in multiple aspects of their lives, such as completing every-day activities around the home, participation in sports, and performance in school [3]. Children with ADHD are less likely to participate in the community and often have a negative stigma from teachers and peers due to the variety of associated behavioral problems [3]. Each child with ADHD can present with varied symptoms and severities that alter the impact of the disorder on the child's participation in daily activities [2].

Children with ADHD present with baseline levels of norepinephrine significantly lower than those without ADHD and have significantly fewer increases in catecholamine dopamine with exercise versus controls without ADHD [4]. To address the inherent lack of dopamine and norepinephrine in children with ADHD, stimulant medication is often prescribed to raise baseline levels [5]. While stimulant medications have been shown to enhance participation and success in the school setting, in some instances, medication may not be the best option. In a review of studies, Poulton reported that 9 out of 21 studies showed decreased growth in children taking stimulant medications as a treatment. Further in that review, two studies showed that children went through rebound growth during "drug holidays," suggesting that their growth was stunted while actively taking medication [6]. Van de Loo-Neus and colleagues hypothesized that the reported growth decline could be a result of decreased appetite, another side effect of the medication [7]. In addition to these common side effects, up to $30 \%$ of children have symptoms that are either not addressed by medication administration or are unable to tolerate the side effects of pharmacotherapy interventions [5]. Additionally, medications are shown to be less effective in treating children who have certain comorbid internalizing disorders, including anxiety or depression, when compared to alternative methods such as physical activity [8]. New research is suggesting that the effect of exercise on motor control of children with ADHD is comparable to the effect of medication [9].

Physical activity has been shown to improve the same catecholaminergic systems that stimulant medications target, thus helping to elevate baseline dopamine and norepinephrine levels, allowing children to mitigate the physiological deficits from ADHD [5]. When Hoza compared aerobic exercise and sedentary classroom activities, they found aerobic exercise had a greater impact on improving attention and behavior $[10,11]$. In another study, Verret showed children who participated in the physical exercise group had significantly lower anxiety-depression scores, fewer social problems, and had improved scores in auditory and sustained attention compared to a control group [3]. In addition, Archer and colleagues reported that exercise had shown benefits in decreasing stress, anxiety, depression, and poor impulse control with improved executive functioning [12]. Further, this study found physical activity increases brain-derived neurotrophic factor, which is an important aspect of normal brain development commonly found to be reduced in children with ADHD [12].

To facilitate healthy development, mental health counselors have started to integrate physical 
activity into their treatment sessions with children with ADHD. Specific techniques include adventure-based counseling, which focuses on physical activity and places a child in a more natural environment. This type of counseling was developed to make interventions more dynamic and action-oriented [13]. Portrie and colleagues described adventure-based counseling as a method to promote feelings of belonging, significance, and perception of self-worth, all of which are potential limitations in children with ADHD [13]. These authors hypothesized that children with ADHD are typically more receptive to this active and adventure-based type of treatment, as it is engaging and can work into their strengths rather than focusing on their limitations [13]. In addition, this treatment used the high energy and attention-seeking behaviors of children with ADHD in a productive way [13]. One example of adventure-based counseling that integrates a more active, hands-on, physically, and mentally challenging activity is rock climbing [13].

Rock climbing is a unique form of physical activity that challenges muscular endurance, requires prolonged attention, and assesses planning ability in people of all ages. There are various forms of rock climbing that can be completed indoors in a controlled environment, two of which are called top roping and bouldering. Top rope climbing involves the climber being in a harness and tied to a rope that goes through a pulley at the top of the wall. The rope is then fed through a belay system that anchors the rope to a partner on the floor to prevent falls. Bouldering, on the other hand, is performed without a rope or harness, and the routes are closer to the ground. Crash pads are placed under the climber to prevent injury with falls/dismounts [14]. Rock climbing can be an activity for people of varying ages, and bouldering has been shown to be a legitimate mode of exercise for elementary aged children [15]. Due to the creativity and decision-making needed to choose a route while rock climbing, Kamijo reported that it challenges the climber to use executive functioning throughout the climb [16]. Kennison found that rock climbing provided a structured learning experience with fear as a motivating factor to help maintain their attention in children with Attention-Deficit Disorder [17]. Additionally, a study by Tower demonstrated a relationship between the sport of rock climbing and the development of problem-solving, executive functioning, planning, decision-making, and learning from mistakes [18]. Previous research of cognitive function in children with cerebral palsy and rock climbing interventions have been inconclusive to date [19].
Based on the neurophysiological effects of exercise, combined with executive function/ problem solving benefits, rock climbing could have a positive impact on the daily function and participation of children with ADHD. Rock climbing could be implemented as a supplement or alternative to medication, helping to reduce harmful side-effects of medication and elicit a positive impact on those exhibiting symptoms associated with ADHD. Although there is no current research on rock climbing for children with ADHD, there is preliminary evidence to support the use of climbing in children with Autism Spectrum Disorder (ASD). Within this study, although the authors did report that climbers working at higher intensity levels demonstrated improved attention test scores, there were no statistically significant differences observed in attention tests with moderate exercise intensity. However, all the parents of participants in this study strongly agreed or agreed that rock climbing had a positive effect on their child $[20,21]$. The purpose of this pilot study was to explore the effects of rock climbing on attention in children with ADHD.

\section{Materials and Methods}

The protocol was reviewed and approved by the Institutional Review Board at Lebanon Valley College. All participants and guardians provided informed consent prior to participation.

\subsection{Adaptations for older adults and seniors}

Children with ADHD between the ages of 8-13 were recruited through flyers sent to parents of local schools. Inclusion criteria consisted of parent reports of a formal diagnosis of ADHD, ages 7-13, and parent/guardian consent. Children were provided with free climbing in exchange for their participation. The Vanderbilt ADHD Diagnostic Rating Scale was used to determine the severity and type of ADHD each child exhibited.

The participants included 5 children ( 4 males, 1 female), with a mean age of 10.2 years old. Four of the children were taking some form of medication, including the following: Concerta, Intuniv, Clonidine, and Strattera. Three of the 5 participants had other medical diagnoses, including attention-deficit disorder, mild autism, Tourette's syndrome, and anxiety. Participant demographics can be found in Table 1. 
Table 1 Participant Demographics

\begin{tabular}{|c|c|c|c|c|c|}
\hline $\begin{array}{c}\text { Participant } \\
\text { Number }\end{array}$ & Age & Gender & Medical Diagnosis & Medication & Vanderbilt Category \\
\hline 1 & 8 & F & ADHD & None & Anxiety/Depression \\
\hline 2 & 12 & M & ADHD, ADD & Concerta & $\begin{array}{c}\text { Inattentive/ } \\
\text { Oppositional Defiant }\end{array}$ \\
\hline 3 & 13 & M & $\begin{array}{c}\text { ADHD, Anxiety, } \\
\text { Tourette's Syndrome }\end{array}$ & Clonidine, Strattera* & $\begin{array}{c}\text { Anxiety/Inattentive/ } \\
\text { Oppositional Defiant }\end{array}$ \\
\hline 4 & 10 & M & $\begin{array}{c}\text { ADHD, Mild Autism, } \\
\text { Anxiety }\end{array}$ & Concerta 27 mg daily & Oppositional Defiant \\
\hline 5 & 9 & M & ADHD & $\begin{array}{c}\text { Allegra/singulair, Concerta } \\
\text { (AM), Intuniv (PM) }\end{array}$ & Does Not Qualify \\
\hline
\end{tabular}

* Participant changed from Clonidine to Strattera with two weeks remaining in the study.

\subsection{Materials}

A harness was donned on each climber prior to starting each session for safety and utilization of the belay system. Handheld stopwatches were used to measure the amount of time the climber spent on the wall during a climb. Nonin pulse oximeters were used to determine the resting heart rate and working heart rate of each child throughout their climbing sessions.

The Trail Making Test (TMT) was used to track progress in attention, planning, and executive functioning. The TMT is one of the most popular neuropsychological tests and was chosen based on the ease of administration and correlation to prior research related to the dependent variable in this study [20]. Trail Making Test B (TMT-B), utilized in the study, required the participant to draw a line connecting numbers and letters in ascending order (1-A-2-B-3-C, etc.). The trail making test provided information on visual search, scanning, speed of processing, mental flexibility, and executive functions of the participant [22]. The TMT-B was administered 2 times during the session: once prior to the onset of climbing, and once at the conclusion of climbing. Composite index reliability for the TMT-B is .92. The test has also shown to exhibit excellent convergent and divergent validity for examining executive functioning [23].

The Conner's' Parent Rating Scale (CPRS) was used as a parental reported assessment tracking behavioral changes of participants over time. This test was administered twice, once prior to the start of the climbing program, and once following the conclusion of the 8-week program. The CPRS is brief and uses the parent's perceptions that results in a reliable and accurate measure of the child's behavior. The reliability of the CPRS in our population is .85-.94 and demonstrates concurrent validity with other behavioral outcome measures such as the Child Behavior Checklist and the Behavior Problem Checklist [24]. Additionally, the CPRS has demonstrated good sensitivity and specificity through discrimination of those with behavioral disorders and in those who are typical [25, 26]. The parents rated their child's behavior using descriptors for a list of behaviors commonly seen in children with ADHD. For the purpose of this research, the overall change in score was more important than the clinical significance of the score, as this threshold will vary depending on the child [26].

The Intervention Rating Profile (IRP-15) was used to determine the social validity and effectiveness of the intervention. The threshold of an "acceptable intervention" has been found to be a score of greater than 52.5 [27]. The IRP-15 was completed by each child's parent at the end of the climbing program, allowing them to share results they had seen with their child. Reliability for the IRP-15 is .98 and validity is .86 based on Cronbach's Alpha [28].

A parent questionnaire, including information about perceived benefits of the study, participant enjoyment, and recommendations for future studies, was provided to parents at the end of the study with an envelope for easy return. Parents were asked to mail back the completed form within the following week. 


\subsection{Design}

This study employed a mixed methods design where both quantitative and qualitative data were collected. Participants were randomly paired with researchers. Independent variables of this study included climbing time and route difficulty. Dependent variables included executive functioning, attention to task, and energy expenditure. Throughout the study, the rock-climbing gym was open to the general public. Extraneous variables included other climbers outside of the study who attended the rock-climbing gym.

\subsection{Procedure}

Prior to the beginning of the study, parents were informed of their own responsibilities and their child's responsibilities throughout the study. Parents of the participants completed the Vanderbilt ADHD Diagnostic Rating Scale at the beginning and the end of the program in order to track changes in the severity and type of ADHD. Parents also assessed their child using the CPRS to establish a baseline behavioral description for each participant.

The climbing program occurred one night per week for eight weeks. Each session lasted 60 minutes in duration. Within each session, participants were taken to a quiet area, helping to minimize distractions, and completed a timed pre-intervention and post intervention Trail Making Test. In addition, at the beginning of each session, each participant's resting heart rate (HR) was taken using the Nonin Pulse Oximeter. The participants were then assisted by the researchers in donning the harness. Each child chose to either top rope or boulder, as well as which routes, he/she wished to perform during the session. Participants were able to switch between bouldering and top-roping as they desired.
Early in the program, participants would climb freely along routes of their choosing. The planned routes that were marked by colored tape changed multiple times during the 8 weeks. At week 4 , each participant was encouraged by the researchers to follow the marked routes and create a plan prior to beginning the climb. Each climb was timed by the researcher. Researchers provided verbal cues to participants when they struggled with a climb, or when the climber asked for help. At the end of the route, researchers took the participant's working HR. At the end of the session, the researcher calculated the average working heart rate and total time the participant was on the rock-climbing wall. Maximum heart rate (HRmax) based on age was calculated with the Astrand equation, and the HRR method was used to find the average intensity of cardiovascular exertion of each climber during the session (Figure 1) [29].

At the end of the program, parents completed the Vanderbilt ADHD Diagnostic Rating Scale and the CPRS. Parents also completed the IRP-15 and a brief survey about the effects of the program. Parents mailed these forms back to the researchers for analysis.

\subsection{Data Analysis}

The Wilcoxon-Signed Ranks Test was used to determine if statistically significant differences existed between pre/posttest for behavioral and attention tests. The SPSS version 23 was used for all statistical analyses. The $P$ value was set at $p<0.05$. Descriptive statistics were used to analyze the demographic data and IRP-15. Open ended responses to the parent questionnaire were read and reread by researchers to identify themes among the responses.

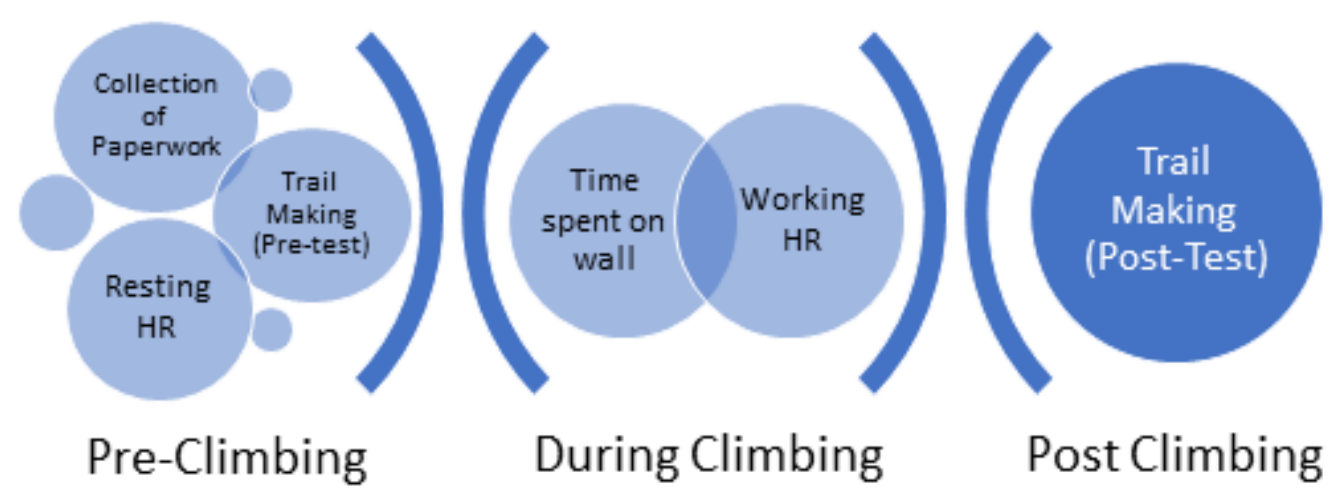

Figure 1 - Procedure of climbing program and data collection performed each week 


\section{Results}

\subsection{Heart Rate and Working Intensity}

Resting heart rate, working heart rate, maximum predicted heart rate, and intensity of effort based on average working heart rate are displayed in Table 2. Three participants were working at a light intensity (30-40\% HRR), and 2 participants were working at a moderate intensity (40-60\% HRR).

Each climber would try both bouldering and top roping within the session; however, climbers 1, 4, and 5 preferred top roping weekly (spent $>50 \%$ top roping), while climbers 2 and 3 spent $>50 \%$ of the session bouldering.

\subsection{Executive Functioning}

Pre and post-test means of the Trail Making Test-B (TMT-B) were compared using the Wilcoxon Signed Ranks test. Statistically significant differences were observed $(p=0.043)$. Specific improvements in measures of executive functioning for each climber can be found in Table 3.

\subsection{Parent Report of Symptoms - Conner's Parent Rating Scale (CPRS)}

CPRS was the quantitative measure used to allow parents to report a change in behavior before and after the climbing intervention. A lower score indicated improved management of symptoms of inattention, hyperactivity, or emotion that are related to ADHD.
Statistically significant improvements were not observed on the Wilcoxon Signed Ranks test $(p=0.066)$. While statistical significance was not observed, visual analysis of the data revealed that $4 / 5$ participants improved from pre to post-test, while one participant remained the same.

\subsection{Post-Test Summary}

The parents of the children who participated in the climbing study were asked to provide feedback to the researchers on the observed improvements, benefits, and challenges associated with the climbing program. All parents indicated that climbing was a beneficial program for their child, as observed in Figure 2. Common themes identified among responses included: perseverance, confidence, and improved problem-solving development over the course of the intervention.

\subsubsection{Perseverance}

Most parents commented on the skills their children learned related to perseverance in order for their child to succeed with climbing. Three of the 5 parents believe that rock climbing became a new passion for their climber because of the individual challenge and the nature of the sport of rock climbing. Similarly, a father elaborated on how he "observed that rock climbing required his child to beat frustration with confidence and perseverance on the wall." Additional statements emphasized the importance of finding success in climbing, which ultimately increased motivation for the children to set and obtain their own specific goals.

Table 2 Exercise intensity of climbing measured by HR before and after activity in BPM

\begin{tabular}{|c|c|c|c|c|c|c|c|}
\hline Participant & $\begin{array}{c}\text { Mean } \\
\text { Resting } \\
\text { HR }\end{array}$ & $\begin{array}{c}\text { Mean } \\
\text { Working } \\
\text { HR }\end{array}$ & $\begin{array}{c}\text { Average } \\
\text { Change }\end{array}$ & $\begin{array}{c}\text { Max } \\
\text { HR }\end{array}$ & $\begin{array}{c}\text { Intensity of } \\
\text { Climbing }\end{array}$ & $\begin{array}{c}\text { Preferred } \\
\text { Method of } \\
\text { Climbing }\end{array}$ & $\begin{array}{c}\text { Category of } \\
\text { Intensity }\end{array}$ \\
\hline 1 & 77.86 & 124.84 & 46.98 & 209.88 & .36 & Top Roping & Light \\
\hline 2 & 83.00 & 139.00 & 56.00 & 206.52 & .45 & Bouldering & Moderate \\
\hline 3 & 97.13 & 153.1 & 56.17 & 205.68 & .52 & Bouldering & Moderate \\
\hline 4 & 88.90 & 123.20 & 34.30 & 208.20 & .29 & Top Roping & Light \\
\hline 5 & 109.70 & 135.40 & 25.70 & 209.04 & .26 & Top Roping & Light \\
\hline Average & 91.32 & 135.15 & 43.83 & 207.86 & .38 & & \\
\hline
\end{tabular}


Table 3 Trail Making Test measured by time to complete in seconds and Conner's' Parent Rating Scale

\begin{tabular}{|c|c|c|c|c|}
\hline Participant & $\begin{array}{c}\text { Mean Pre-Trail } \\
\text { Making (s) }\end{array}$ & $\begin{array}{c}\text { Mean Post Trail } \\
\text { Making (s) }\end{array}$ & $\begin{array}{c}\text { Pre-test } \\
\text { CPRS }\end{array}$ & $\begin{array}{c}\text { Post-test } \\
\text { CPRS }\end{array}$ \\
\hline 1 & 73.67 & 70.73 & 9 & 0 \\
\hline 2 & 58.60 & 56.60 & 9 & 7 \\
\hline 3 & 120.75 & 80.78 & 23 & 14 \\
\hline 4 & 92.10 & 72.40 & 8 & 8 \\
\hline 5 & 52.12 & 36.60 & 17 & 11 \\
\hline Average & 79.45 & 63.42 & 13.2 & 8.0 \\
\hline
\end{tabular}

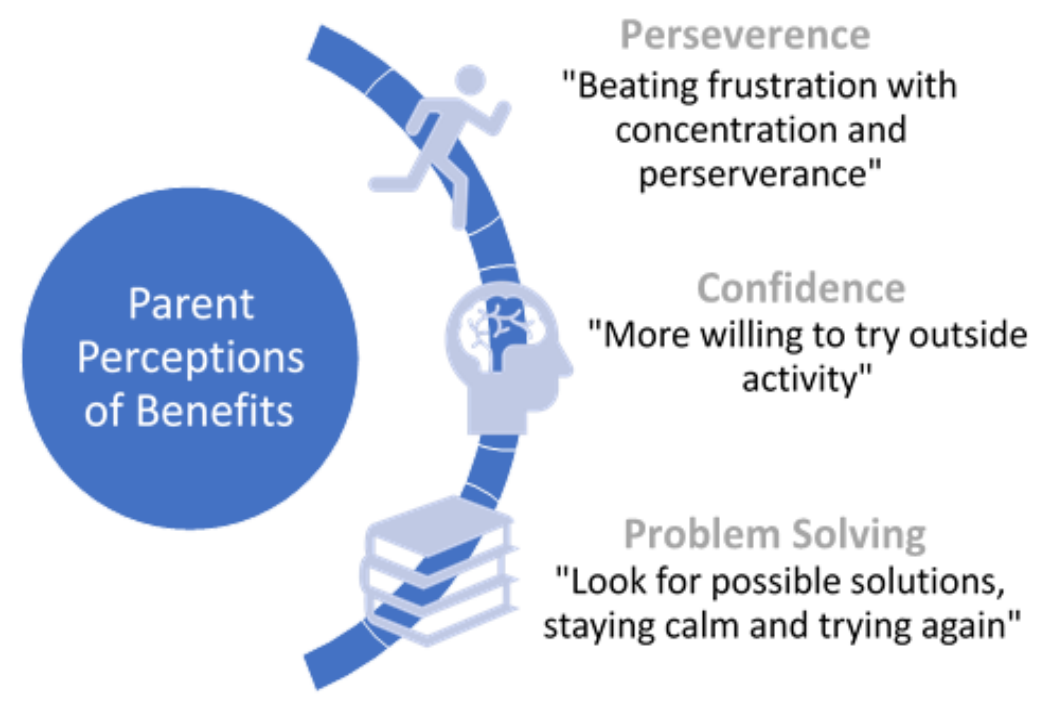

Figure $\mathbf{2}$ - Common themes from parents' perceived benefits of climbing program

\subsubsection{Confidence}

Regarding the individual challenge of rock climbing, one mother stated her son could build trust by having the same person working with him every week. Each parent believed that the program made the climbers more confident in themselves because they were able to overcome their fears and perform things they previously thought they could not. A different parent added to this observation by saying, "[their] climber was more self-confident and is more willing to do outside activities." Additionally, each parent commented on the positive impact the instructors had on their climbers by noting them to be encouraging and supportive, which allowed them to create new relationships to build the confidence of each climber.

\subsubsection{Problem Solving}

Additionally, all 5 parents believed their climber improved in their ability to plan, set goals and problem solve. One parent noticed that "[their] climber learned how to problem solve and persevere during high stress situations which were accomplished by looking at all possible solutions, staying calm and trying again." Additionally, another parent stated, "[they] are trying to use the skills of problem solving in rock climbing in other situations they come across. [They] try to refer back to climbing because it is something the child is familiar with and they have experienced success with problem solving on the wall." The individual aspect of rock climbing was important to 2 of the 5 parents because their child would often get distracted in team sports as there is so much extraneous activity happening at one time. Additionally, the climber can 
work towards their own specific goals with the assistance of the instructor. Each climber was challenged to problem solve individually to improve independence while overcoming their own personal obstacles.

\subsection{IRP-15}

The average score on the IRP was 86.3 , which is above the cut-off score of 52.5 that would indicate rock climbing as an effective intervention. The IRP-15 showed $100 \%$ of the parents of participating climbers believed this was an effective intervention to manage the symptoms of their children. Of the 4 responses received, all 4 parents strongly agreed that the activity was a good way to handle their child's participation needs and a fair way to engage their child in the community. They each either agreed or strongly agreed with the statement that rock climbing is an acceptable and appropriate activity for a child with ADHD. Similarly, all parents strongly agreed that they would suggest this activity to other parents.

\section{Discussion}

This pilot study examined if participation in rock climbing for children with ADHD led to improved attention and behavior. Based on the results, 3 out of the 5 children were exercising at a light intensity of only $30-40 \%$ of their heart rate reserve. After visual analysis of the climbing tendencies of these 3 children, it was found that these children typically chose top rope climbing each week. The other 2 children exercised at a moderate intensity of $40-60 \%$ of their heart rate reserve. These 2 children typically chose bouldering as their rock-climbing style of choice.

There are many reasons that the children who chose bouldering may have been working at a greater intensity. These children may have worked harder because they did not have the external support of the harness to hold themselves on the wall. If they could not support themselves, they would have to start again from the bottom. Additionally, when bouldering, children performed more powerful moves for a shorter amount of time. Bouldering allowed children to exert themselves further because bouldering had shorter routes than top roping. This concept is consistent with the literature, as Fend et al. reported that bouldering is a legitimate mode of exercise for elementary aged children [15]. Despite this difference in exercise intensity, all the participants in the climbing program demonstrated improvement in their attention and behavior.
Exercise intensity while climbing may vary based on the patient population. Oriel et al., found that children with ASD demonstrated higher levels of exertion while exclusively top roping [20], which is inconsistent with this study's findings. This inconsistency may be explained by the level of executive functioning and planning encouraged by participants with ADHD while top roping in this study. The participants with ADHD, in this study, often took rest breaks while top roping when they were stuck, and they took time to plan the most efficient route. In Oriel's study, the subjects with ASD may have used less executive planning while climbing because they were not instructed to follow specific routes. Therefore, the climbers took fewer rest breaks, completed their climbs more quickly, and were able to exercise at a higher intensity level [20]. More research is needed on the effects of top roping on cardiovascular expenditure due to the varied congruence between the results of this study and past studies with similar measures.

The outcomes for this study were positive when looking at attention using TMT-B, as there were significant changes between pre and post climbing attention. These results show that rock climbing correlates with improving executive functioning. Rock climbing could also be used throughout the day to improve the attention of a child. Further, the improvements on the TMT-B demonstrate increases in attention among all participants in the study. However, there were multiple factors that may have affected the results of the test, including education level, understanding of the task, cues required, and memorization. At the beginning of the study, researchers noted difficulty with comprehension of the directions for the TMT-B, as most climbers required cueing to complete correctly. The decrease in time of completion could be attributed to a learning curve and potential memorization of the correct pattern. Despite the conditions around the TMT-B, there were significant findings for intrasession attention improvement, suggesting that climbing was a purposeful intervention for the participants within this study.

The CPRS quantified the behavioral changes of participants throughout the duration of the climbing program. There were no statistically significant improvements $(p=.066)$ observed based on the Wilcoxon Signed Ranks Test. These results may have been due to the small sample size. With a visual analysis of the data, 4 out of 5 of the participants 
improved between the pre and post-test. While there is no identified minimal detectable change for the CPRS, the trend of decreased scores over time indicated that the climbers improved in management of conduct problems, learning problems, psychosomatic factors, impulsive/hyperactive behaviors, and anxiety. The questions on the CPRS were subjective and based solely on the performance of the child from the perspective of the parent. This style of questioning introduced an element of bias into the scoring, but the subjective scores with the CPRS were consistent with the qualitative information collected at the end of the study.

All parents identified benefits of the climbing program, which included perseverance, problem solving, and elevated confidence. In addition to the parent's perspective of change, the research team observed an increase in motivation, acceptance of challenges, and improved independence that grew over the eight-week intervention. Rock climbing appeared to make a positive impact on the families and lives of these 5 participants.

The IRP-15 results demonstrated the utility of rock climbing as an effective intervention for symptoms of $A D H D$, according to parent report. With the average score of each test greater than the standardized cut-off score, it is reasonable to conclude that the participating parents found value in rock climbing, and they would find this activity an acceptable form of intervention for their own child, while similarly recommending it to other parents. It was noted that one child did not return this form which limited the amount of data collected in this aspect of the study. However, the results of this IRP-15 survey supported the qualitative responses from each of the parents to indicate the utility of skills learned in rock climbing in daily activity and participation.

Further limitations to this study included a small sample size, variable accountability of the climbers, and inconsistent paperwork return, which impacted the accuracy of data collection. Consistency within the session could also have been improved with a standardized cueing protocol that was similar between climbers. In future studies, a progression of the climbing program should be standardized amongst all climbers while adjusted based on climbing ability. Additionally, some climbing routes were too challenging for the smaller participants, and the availability of climbing space was variable due to extraneous people in the environment that were also distracting to the climbers.
The qualitative and quantitative results from this study suggested that rock climbing can improve planning and short-term attention in children with ADHD. These skills developed by the children can be used at home and in school. The qualitative results of this study supported research by Hoza and Archer, which suggested that physical activity improves behavior and attention in children with ADHD $[10,11]$. Based on parent report, children can transfer the knowledge and skills gained during rock climbing into their daily lives. This skill carryover could also be used in the school setting when students are faced with classroom problems. Further, parents could prompt their child to use these newly developed problemsolving methods at home to improve behavior. In addition, the quantitative results of this study supported the research of Tower and Kamijo, reporting that participants have improved executive functioning after rock climbing $[18,16]$. This research could be beneficial for elementary-aged children with ADHD who struggle in the classroom. By taking breaks for physical activity (i.e. rock climbing, recess, etc.), students could facilitate improved attention and behavior during instructional education. Teachers could orient their daily schedule to teach more challenging subjects after a physical activity break to foster improved attention and learning. This research supports the public movement for elementary schools to encourage daily physical exercise through scheduled activity periods in order to improve attention and manage behavior of children with ADHD [5].

\section{Conclusion}

ADHD is a common disorder that negatively impacts children, leading to inattention, impulsivity, and hyperactivity. These traits impact children's abilities to stay involved with their peers, complete school-based activities, and participate in the community. Many children attempt to control their symptoms by taking prescription medications; however, these medications often cause multiple sideeffects that hinder children in their everyday lives. This pilot study suggested that participation in a community-based rock-climbing program may lead to improvements in attention. Therefore, climbing should be considered as an intervention to improve attention in children with ADHD.

\section{References}

[1] Attention-Deficit / Hyperactivity Disorder (ADHD). Centers for Disease Control and 
Prevention

https://www.cdc.gov/ncbddd/adhd/data.html

Published September 21, 2018. Accessed September 26, 2018.

[2] Symptoms of ADHD. ADHD Institute. https://adhd-institute.com/assessment-diagnosis Published September 21, 2018. Accessed September 26, 2018.

[3] C. Verret, M. Guay, C. Berthiaume, P. Gardiner, \& L. Béliveau, A Physical Activity Program Improves Behavior and Cognitive Functions in Children With ADHD, Journal Of Attention Disorders, 16(1) (2012) 71-80. DOI | PubMed

[4] S. Wigal, D. Nemet, J.M. Swanson, R. Regino, J. Trampush, M.G. Ziegler, \& D.M. Cooper, Catecholamine response to exercise in children with attention deficit hyperactivity disorder, Pediatric Research, 53(5) (2003) 756-761. DOI I PubMed

[5] S. Wigal, N. Emmerson, J. Gehricke, and P. Galassetti, Exercise: Applications to Childhood ADHD, Journal of Attention Disorders, 17(4) (2012) 279-290. DOI I PubMed

[6] A. Poulton, Growth on stimulant medication; clarifying the confusion: a review, Archives of Disease in Childhood, 90(8) (2005) 801-806. DOI I PubMed

[7] G.H.H. van de Loo-Neus, N. Rommelse, \& J.K. Buitelaar, To stop or not to stop? How long should medication treatment of attention-deficit hyperactivity disorder be extended?, European Neuropsychopharmacology, 21(8) (2011) 584599. DOI | PubMed

[8] N. Al Ghriwati, J. Langberg, W. Gardner, J. Peugh, K. Kelleher, \& R. Baum, W.B. Brinkman, P. Lichtenstein, J.F. Epstein, Impact of Mental Health Comorbidities on the Community-Based Pediatric Treatment and Outcomes of Children with Attention Deficit Hyperactivity Disorder, Journal of Developmental \& Behavioral Pediatrics, 38(1) (2017) 20-28. DOI I PubMed

[9] R. Vysniauske, L. Verburgh, J. Oosterlaan, M. Molendijk, The Effects of Physical Exercise on Functional Outcomes in the Treatment of ADHD: A Meta-Analysis, Journal of Attention Disorder, 24(5) (2020) 644-654. DOI I PubMed

[10] B. Hoza, C. Martin, A. Pirog, \& E.K. Shoulberg, Using Physical Activity to Manage ADHD Symptoms:The State of the Evidence, Current Psychiatry Reports, 18(12) (2016) 113. DOI I PubMed
[11] B. Hoza, A.L. Smith, E.K. Shoulberg, K.S. Linnea, T.V. Dorsch, \& J.A. Blazo, C.M. Alerding, G.P. McCabe, A Randomized Trial Examining the Effects of Aerobic Physical Activity on AttentionDeficit/Hyperactivity Disorder Symptoms in Young Children, Journal of Abnormal Child Psychology, 43(4) (2016) 655-667. DOI । PubMed

[12] T. Archer, \& R. Kostrzewa, Physical Exercise Alleviates ADHD Symptoms: Regional Deficits and Development Trajectory, Neurotoxicity Research, 21(2) (2011) 195-209. DOI I PubMed

[13] T. Portrie-Bethke, N. Hill, J. Bethke, StrengthBased Mental Health Counseling for Children with ADHD: An Integrative Model of Adventure-Based Counseling and Adlerian Play Therapy, Journal of Mental Health Counseling, 31(4) (2009) 323-337. DOI

[14] Bridgeman G. Indoor (Gym) Climbing Basics. REI Co-op Journal, https://www.rei.com/learn/expert-advice/indoorclimbing.html Accessed September 3, 2018.

[15] M. Fend, J. Muras, J. Steffen, R. Battista, \& A. Elfessi, Physiological Effects of Bouldering Activities in Upper Elementary School Students. Physical Educator, 68(4) (2011) 199-209.

[16] K. Kamijo, \& Y. Takeda, Regular physical activity improves executive function during task switching in young adults, International Journal of Psychophysiology, 75(3) (2010) 304-311. DOI I PubMed

[17] J.A. Kennison, R. Watters, (1996), Therapy in the Mountains. Proceedings of the 1995 International Conference on Outdoor Recreation and Education.

[18] A. Tower, Get your climb on, Yoga Journal, (263) (2014) 56-63.

[19] M. Schram Christensen, T. Jensen, C.B. Voigt, J.B. Nielsen, J. Lorentzen, To be active through indoor-climbing: an exploratory feasibility study in a group of children with cerebral palsy and typically developing children. BMC neurology. 17(1) (2017) 112. DOI I PubMed

[20] Kathryn N. Oriel, Jennifer Wood Kanupka, Adam T. Fuehrer, Kayla M. Klumpp, Katelyn N. Stoltz, Daniel W. Willey \& Michael L. Decvalcante, The Impact of a Rock Climbing Program for Adolescents with Autism Spectrum Disorder: A Pilot Study, International Journal of Kinesiology in Higher Education, 2(4) (2018) 113-126. DOI 
[21] Q. Ng, C. Ho, H. Chan, B. Yong, \& W. Yeo, Managing childhood and adolescent attentiondeficit/hyperactivity disorder (ADHD) with exercise: A systematic review, Complementary Therapies In Medicine, 34 (2017) 123-128. DOI | PubMed

[22] T. Tombaugh, Trail Making Test A and B: Normative data stratified by age and education, Archives of Clinical Neuropsychology, 19(2) (2004) 203-214. DOI I PubMed

[23] C. Reynolds, Comprehensive Trail Making Test (CTMT), Archives Of Clinical Neuropsychology, 19(5) (2004) 703-708. DOI

[24] C.K. Conners, G. Sitarenios, J.D.A. Parker, J.N. Epstein, The revised Conners' Parent Rating Scale (CPRS-R): factor structure, reliability, and criterion validity, Journal of Abnormal Child Psychology, 26(4) (1998) 257-268. DOI । PubMed

[25] Margot Prior, Anne Leonard, Glenice Wood, A Comparison Study of Preschool Children Diagnosed as Hyperactive, Journal of Pediatric Psychology, Volume 8(2) (1983) 191-207. DOI

[26] C.K. Conners, (2001). Conners rating scalesrevised. Toronto: Multi-Health Systems.

[27] S.N. Elliott, \& M.V.B. Treuting, The behavior intervention rating scale: Development and validation of a pretreatment acceptability and effectiveness measure, Journal of School Psychology, 29(1) (1991) 43-51. DOI

[28] C.A. Finn, \& I.E. Sladeczek, Assessing the social validity of behavioral interventions: A review of treatment acceptability measures, School Psychology Quarterly, 16(2) (2001) 176-206. DOI

[29] ACSM's Guidelines for Exercise Testing and Prescription. 9th ed. Baltimore, MD: Lippincott Williams Wilkins, 2014.

\section{Acknowledgement}

This research is supported by Climbnasium in Mechanicsburg, PA who donated time and space in their climbing gym for this study. Lebanon Valley College provided other tools necessary to complete tests and measures. In addition to the listed authors, Kelsey Payung PT, DPT participated in data collection. No direct funding was accepted for this study

\section{Funding}

Nil.

\section{Authors Contribution}

Individual contributions are as follows: All authors participated in the creation of protocol and study design. JP led participation recruitment. EA, GM, KDAC, RMD led weekly climbing sessions with participants and collected relevant data. JP and KO collaborated to analyze data. All members assisted in writing up manuscript. EA and $\mathrm{KO}$ adapted this manuscript for Journal of Sport and Health Science.

\section{Ethics Approval}

This study was approved by Institutional Review Board at Lebanon Valley College.

\section{Conflict of interest}

Nil

Availability of data and material No additional data are available

\section{Informed consent}

All participants and guardian provided informed consent prior to participation

\section{About the License}

(c) The author(s) 2020. The text of this article is open access and licensed under a Creative Commons Attribution 4.0 International License 